\title{
Metabolism in the Gastrointestinal Tract: Relevance for Drug Development
}

For orally administered drugs, metabolism in the gastrointestinal tract (GIT) can decrease the fraction of the dose bioavailable in the systemic circulation (e.g. by firstpass metabolism), but in the case of prodrugs also enable the pharmacologically active moiety to become bioavailable at all. The role of the liver in drug metabolism is widely known and thoroughly investigated. But also the GIT can have a significant role in drug metabolism. The purpose of this paper is to describe these aspects along the consecutive segments of the GIT, i.e. mouth, esophagus, stomach, small intestine, and large intestine.

\section{Mouth}

The oral cavity and pharynx are the first parts of the body a medicine encounters when taken orally. Several drug-metabolizing enzymes are located in the buccal epithelial cells, such as oxidases (e.g. cytochrome P450), reductases and cyclooxidases [1]. These are, however, far less pronounced than in the liver, for example. Furthermore, an immediate-release tablet taken orally likely does not disintegrate in the mouth but is swallowed quickly. Therefore, an interaction between the oral cavity or pharynx and the drug is not to be expected. Exceptions to this are special formulations like orally disintegrating tablets (ODT), sublingual tablets, buccal tablets, sprays, or solutions. Formulations that release drugs already in the mouth can be used for local or systemic therapy, the latter bypassing first-pass metabolism $[2,3]$.

\section{Esophagus}

After swallowing, a perorally administered drug passes through the esophagus, which is approx. $25 \mathrm{~cm}$ long and $2 \mathrm{~cm}$ in diameter [4]. Due to the fast transit time of a drug and the physiological characteristics of the esophagus with its poor blood supply and squamous epithelium, absorption and metabolization of the drug is not to be expected [5]. However, local therapy approaches, e.g. suspensions for eosinophilic esophagitis, are used and there are also examples of local esophageal damage caused by solid oral drugs like bisphosphonates when taken in the supine position or with too little fluid [6,7].

\section{Stomach}

No significant drug absorption or metabolization takes place in the stomach. The physiochemical conditions in the stomach can, however, impact the drug dissolution and lead to drug degradation processes, e.g. due to acid instability (e.g. proton pump inhibitors). Due to its mainly acidic conditions, the stomach is sparsely populated with bacteria [8]. The gastric emptying determines the rate at which drugs reach the small intestine.

\section{Small intestine}

The small intestine is approx. 3-6 m long and has by far the largest surface area of the GIT due to its histology comprising villi and microvilli. It is divided from proximal to distal into duodenum, jejunum, and ileum. One of its main physiological tasks is the absorption of nutrients from food. Drugs are also mostly absorbed through the small intestine. In recent years, the importance of the small intestine, along with the liver, as an organ important for drug metabolism has been increasingly discussed. Phase I as well as phase II metabolization reactions were observed in the small intestine [9]. Many of the drug-metabolizing enzymes that are localized in the liver (e.g. cytochromes P450, UDP-glucosyltransferases and -sulfotransferases) can also be found to a lesser extent in the small intestine [10]. The distribution and activity of these enzymes decreases from the proximal to the distal small intestine [11]. As in the liver, the metabolism of a drug before it reaches the systemic circulation is described as first-pass metabolism. If the drug administered is not a prodrug that is converted into its active form by metabolization, pronounced first-pass metabolism results in reduced bioavailability.
Paine et al. investigated the contribution of the small intestine to the first-pass metabolism of the CYP3A4 substrate midazolam as early as 1996 [12]. For this purpose, they administered midazolam intraduodenally to 5 patients and intravenously to 5 patients after removal of the liver during liver transplantation. Subsequently, arterial and hepatic portal venous blood samples were collected simultaneously in the anhepatic phase. After reperfusion of the donor liver, arterial blood samples were further collected. The mean extraction rates of the two groups were compared and the authors were able to show that the small intestine significantly contributes to the first-pass metabolism of midazolam. This can be relevant for drug development when it comes to the treatment of patients with impaired liver function, for example.

In contrast to the effect of first-pass metabolism, a drug that is subject to a pronounced enterohepatic circulation may show an increased bioavailability. The enterohepatic circulation describes the circulation of drugs through absorption (mostly in the small intestine), possibly Phase II metabolization in the liver (e.g. conjugation) and subsequent reintroduction into the small intestine through the bile and bile ducts, and reabsorption. In case of drugs re-entering the small intestine conjugated, enteral bacteria convert it back to the parent compound before reabsorption. This circulation can occur several times a day and thus contribute to longer residence times of the drug in the body (e.g. digitoxin) [13].

Similar to the stomach, the proximal small intestine is sparsely populated with bacteria. In the course from the distal small intestine to the large intestine, however, the colonization increases [14]. Thus, the small intestine may contribute to drug metabolism via its microbiome. One example is levodopa, which can partly be converted to dopamine by bacteria in the small intestine and is thus no longer able to pass the blood-brain barrier and unfold its full effect there [15]. With regard to the efficacy of levodopa, large inter-individual variability is reported which is also attributed to 
the variability in the microbiome of these patients. The characteristics of the human microbiome are largely determined by the large intestine, as this has the highest microbiotal colonization of the GIT [8].

\section{Large Intestine}

The large intestine consists of caecum, colon, rectum, and anus and has a total length of about $1.5 \mathrm{~m}$. Its main function is the absorption of remaining water and electrolytes. The colonic bacteria produce certain vitamins (Vitamin B and K) which are subsequently absorbed. The composition of the intestinal microbiome is subject to strong inter-individual variability, as it depends on external factors such as food, but is also influenced by genetic factors [16]. Due to the high level of bacterial colonization of the colon, it may play an important role in the metabolism of drugs. The part of the ascending colon is of particular interest here [17]. Chemical reactions such as reduction, hydrolysis and dehydroxylation are described.

An example of such a reaction is sulphasalazine, which is mainly cleaved by colonic bacteria into 5-aminosalicylic acid (5-ASA) and sulphapyridine by means of azo reduction [18]. This fact is used in the treatment of inflammatory diseases of the colon (e.g. ulcerative colitis), as 5-ASA has an antiinflammatory effect in the colon. Sulphapyridine, on the other hand, has antibiotic properties and is absorbed after reduction and is responsible for a significant part of the systemic side effects. This example shows the potential complexity of these processes in the GIT and underlines the importance to take this into account in the development of a drug.

\section{Consequences for Drug Development}

The metabolic capacity of the GIT described above impacts the bioavailability of many perorally administered drugs. Its pharmacological relevance depends on whether the drug needs to be or just is systemically bioavailable or is locally acting in the GIT. During drug development, these factors need to be investigated under a va- riety of conditions, e.g. concomitant medications, influence of food components, or can be specifically addressed, e.g. with formulations/routes of administration circumventing a first-pass metabolism.

An example of the optimization of a delivery technology is the antiemetic drug prochlorperazine, which was originally only available as an immediate-release oral tablet. To circumvent the pronounced firstpass metabolism of the drug, it was formulated as a buccal oral-release tablet. This resulted in an approximately twofold increase in systemic exposure compared to the immediate-release tablet [2].

Probably the best-known food interaction in the GIT is the interaction of grapefruit juice with drugs. Grapefruit juice (and other fruit juices) inhibits the CYP3A4 enzymes in the enterocytes of the small intestine, but not the liver, which are involved in the metabolization of many drugs. In the example of the calcium channel antagonist felodipine, this can lead to a significant increase in exposure, which can result in reduced blood pressure [19]. Relevant interactions have also been reported for other calcium channel blockers such as nifedipine, but also for other substance classes such as statins. In development, it is important to investigate the occurrence and relevance of the interaction and to derive recommendations to be outlined in the label of the product.

\section{Conflict of Interest}

Joachim Höchel and Maximilian Feldmüller are both employees of Bayer AG and have no conflict of interest to declare.

\section{Authors}

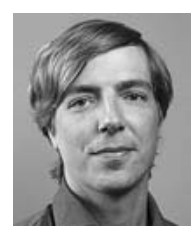

\section{Maximilian Feldmüller}

Bayer AG, Pharmaceuticals Research \& Development, Clinical Pharmacology

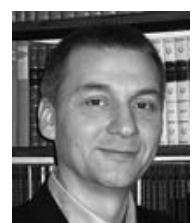

\section{Joachim Höchel}

Bayer AG, Pharmaceuticals Research \& Development, Clinical Pharmacology

\section{Correspondence}

Joachim Höchel

Maximilian Feldmüller

Bayer AG

Research \& Development, Pharmaceuticals

Clinical Pharmacology

13342 Berlin

Germany

Joachim.hoechel@bayer.com

\section{References}

[1] Yamahara H, Lee VHL. Drug metabolism in the oral cavity. Advanced Drug Delivery Reviews 1993; 12: 25-40

[2] Finn A, Collins J, Voyksner R et al. Bioavailability and metabolism of prochlorperazine administered via the buccal and oral delivery route. J Clin Pharmacol 2005; 45: 1383-1390

[3] Smart JD. Buccal drug delivery. Expert Opin Drug Deliv 2005; 2: 507-517

[4] Li Q, Castell JA, Castell DO. Manometric determination of esophageal length. Am J Gastroenterol 1994; 89: 722-725

[5] Batchelor $\mathrm{H}$. Bioadhesive dosage forms for esophageal drug delivery. Pharmaceutical Research 2005; 22: 175-181

[6] Dellon ES, Sheikh A, Speck O et al. Viscous topical is more effective than nebulized steroid therapy for patients with eosinophilic esophagitis. Gastroenterology 2012; 143: 321-324

[7] De Groen PC, Luebbe DF, Hirsch LF et al. Esophagitis associated with the use of alendronate. N Engl J Med 1996; 14: 1016 1021

[8] Macfarlane GT, Macfarlane S. Human colonic microbiota: ecology, physiology and metabolic potential of intestinal bacteria. Scand J Gastroenterol Suppl 1997; 32: 3-9

[9] George CF. Drug Metabolism by the gastrointestinal mucosa. Clin Pharmacokinet 1981; 6: 259-274

[10] Back D], Roger SM. Review: first-pass metabolism by the gastrointestinal mucosa. Aliment Pharmacol Therap 1987; 1: 339-357

[11] Krishna DR, Klotz U. Extrahepatic metabolism of drugs in humans. Clin Pharmacokinet 1994; $26: 144-160$

[12] Paine MF, Shen, DD, Kunze KL et al. First-pass metabolism of midazolam by the human intestine. Clin Pharmacol Ther 1996; 60: 1424

[13] Aldwell JH, Bush CA, Greenberger NJ. Interruption of the enterohepatic circulation of digitoxin by cholestyramine: II. Effect on metabolic disposition of tritium-labeled digitoxin and cardiac systolic intervals in man. J Clin Invest 1971; 12: 2638-2644

[14] Simon GL, Gorbach SL. Intestinal flora in health and disease. Gastroenterology 1984; 86: 174-193 
[15] Rekdal VM, EN, Bisanz JE et al. Discovery and inhibition of an interspecies gut bacterial pathway for levodopa metabolism. Science 2019; 364: eaau6323

[16] Consortium, Human Microbiome Project. Structure, function and diversity of the healthy human microbiom. Nature 2012; 486: 207-214

[17] Sun C, Chen L, Shen Z. Mechanisms of gastrointestinal microflora on drug metabolism in clinical practice. Saudi Pharm J 2019; 8: 1146-1156
[18] Schroder H, Campbell DES. Absorption, metabolism, and excretion of salicylazosulfapyridine in man. Clin Pharmacol Ther 1972; 13: 539-551

[19] Wilkinson G. Drug Metabolism and variability among patients in drug response. $\mathrm{N}$ Engl J Med 2005; 352: 2211-2221 